\title{
2019 Chinese clinical guidelines for the management of hepatocellular carcinoma: updates and insights
}

\author{
Di-Yang Xie ${ }^{1}$, Zheng-Gang Ren ${ }^{1}$, Jian Zhou $^{1,2}$, Jia Fan ${ }^{1,2}$, Qiang Gao ${ }^{1}$ \\ ${ }^{1}$ Liver Cancer Institute, Zhongshan Hospital, Key Laboratory of Carcinogenesis and Cancer Invasion (Ministry of Education), Fudan University, \\ Shanghai, China; ${ }^{2}$ Institute of Biomedical Sciences, Fudan University, Shanghai, China \\ Contributions: (I) Conception and design: All authors; (II) Administrative support: All authors; (III) Provision of study materials or patients: All \\ authors; (IV) Collection and assembly of data: All authors; (V) Data analysis and interpretation: All authors; (VI) Manuscript writing: All authors; (VII) \\ Final approval of manuscript: All authors. \\ Correspondence to: Qiang Gao, MD, PhD. Department of Liver Surgery and Transplantation, Liver Cancer Institute, Zhongshan Hospital, Fudan \\ University, Shanghai 200032, China. Email: gao.qiang@zs-hospital.sh.cn.
}

Importance: Approximately half of newly-diagnosed hepatocellular carcinoma (HCC) cases in the world occur in China, with hepatitis B virus (HBV) infection being the predominant risk factor. Recently, the guidelines for the management of Chinese HCC patients were updated.

Objective: The past decade has witnessed a great improvement in the management of hepatocellular carcinoma (HCC). This study reviews the recommendations in the 2019 Chinese guidelines and makes comparison with the practices from the Western world.

Evidence Review: The updated recommendations on the surveillance, diagnosis, and treatment algorithm of HCC in the 2019 Chinese guidelines were summarized, and comparisons among the updated Chinese guidelines, the European Association for the Study of the Liver (EASL) and the American Association for the Study of Liver Diseases (AASLD) guidelines were made.

Findings: Besides imaging and pathological diagnoses, novel biomarkers like the seven-micro-RNA panel are advocated for early diagnoses and therapeutic efficacy evaluation in the updated Chinese guidelines. The China liver cancer (CNLC) staging system, proposed in the 2017 guidelines, continues to be the standard model for patient classification, with subsequent modifications and updates being made in treatment allocations. Compared to the Barcelona Clinic Liver Cancer (BCLC) system, the CNLC staging system employs resection, transplantation, and transarterial chemoembolization (TACE) for more progressed HCC. TACE in combination with other regional therapies like ablation or with systemic therapies like sorafenib are also encouraged in select patients in China. The systemic treatments for HCC have evolved considerably since lenvatinib, regorafenib, carbozantinib, ramucirumab and immune checkpoint inhibitors (ICIs)were first prescribed as first-line or second-line agents.

Conclusions and Relevances: Novel biomarkers, imaging and operative techniques are recommended in the updated Chinese guideline. More aggressive treatment modalities are suggested for more progressed HBV-related HCC in China.

Keywords: Clinical practice guidelines; hepatocellular carcinoma (HCC); diagnosis; treatment algorithm

Submitted May 10, 2020. Accepted for publication Jun 22, 2020.

doi: $10.21037 / \mathrm{hbsn}-20-480$

View this article at: http://dx.doi.org/10.21037/hbsn-20-480 


\section{Introduction}

Liver cancer is the fourth most common cancer and the second most lethal cancer in China $(1,2)$. Hepatocellular carcinoma (HCC) represents $85-90 \%$ of primary liver cancer and constitutes a major global health problem. The main risk factor for HCC in China is chronic hepatitis $\mathrm{B}$ virus (HBV) infection, whereas HCC in the West is largely attributable to hepatitis $\mathrm{C}$ virus (HCV) infection and alcohol intake. These distinct risk factors contribute to disparities in the genetic landscape, molecular subtype, and clinical phenotypes of HCC cases between China and Western countries $(3,4)$. The neonatal $\mathrm{HBV}$ vaccination program and the antiviral treatment of infected individuals have resulted in a decline in HCC incidence (5-7). Efforts to improve the diagnostic sensitivity and therapeutic efficacy of HCC treatments have contributed to a decrease of $20.3 \%$ in age-standardized mortality in China from 1990 to 2017 (2). In line with typical standards management in Western countries, The European Association for the Study of the Liver (EASL) and the American Association for the Study of Liver Diseases (AASLD) updated the clinical practical guidelines on the management of HCC in $2018(8,9)$. To account for the heterogeneity in the prevalence, phenotype, and treatment responses of HCC between China and other countries, guidelines specific to Chinese patients have been established in kind (10). With the accumulation of new evidence, an updated 2019 version has been recently published (11). Herein, we summarize the updated recommendations on the surveillance, diagnosis, and treatment algorithm of HCC in the 2019 Chinese guidelines, and compare them with the 2018 EASL and the 2018 AASLD guidelines.

We present the following article in accordance with the Narrative Review reporting checklist (available at http:// dx.doi.org/10.21037/hbsn-20-480.

\section{Surveillance and diagnosis}

All patients with liver cirrhosis, irrespective of etiology or country of origin, warrant surveillance (12). Non-cirrhotic patients with chronic viral hepatitis are also recommended for surveillance in China (13). Various risk stratification scoring systems including CUHCC, REACH-B in China, and PAGE-B in Europe, have been developed to discriminate high-risk patients from non-cirrhotic patients with HBV infection (14-16). As one of the most recent EASL guideline revisions, Caucasian patients at intermediate or high risk of HCC as defined by a PAGE-B score $\geq 10$ are now considered candidates for surveillance. Meanwhile, the EASL has extended the surveillance criteria for patients with bridging fibrosis (Metavir F3) from concomitant chronic hepatitis $\mathrm{C}$ to any etiology. Regarding the surveillance mode, ultrasound follow-up every 6 months is unanimously agreed upon, whereas alfa-fetoprotein (AFP) for surveillance testing is obligatory in China, optional in the USA, and not recommended in Europe. Notably, HBVrelated HCCs tend to have a higher AFP level compared to HCC due to other causes (4). As such, AFP plus ultrasound surveillance is associated with an improved curative treatment rate and long-term survival (17).

Diagnosis of HCC can be established by non-invasive imaging criteria and/or pathology. The imaging diagnostic algorithm in the updated Chinese version is consistent with the previous 2017 version: for patients with chronic hepatitis $\mathrm{B} / \mathrm{C}$ or cirrhosis of any etiology, nodules $>2 \mathrm{~cm}$ in diameter can be diagnosed as HCC based on the typical features on one imaging technique, whereas nodules $\leq$ $2 \mathrm{~cm}$ need confirmation by two imaging modalities (10). Dynamic computed tomography (CT) or magnetic resonance imaging (MRI) is accepted as the optimal imaging modality for HCC diagnosis across different guidelines $(8,9,18)$. According to the results of recent meta-analyses, the superiority of dynamic MRI over CT for the diagnosis of HCC $\leq 2 \mathrm{~cm}$ has been acknowledged in the 2019 Chinese guidelines, whereas the two modalities are equally recommended by the AASLD $(19,20)$. The relatively low sensitivity of coincidental CT and MRI for diagnosis of lesions measuring $1-2 \mathrm{~cm}$ has led the EASL and the AASLD to reaffirm the application of a single modality to diagnose lesions $\geq 1 \mathrm{~cm}$ in cirrhotic patients (21). Arterial phase hyperenhancement (APHE) with washout in the portal venous or delayed phases on CT or MRI using extracellular contrast agents, and APHE with washout in the portal venous phase on MRI using gadoxetic acid are listed as typical hallmarks of HCC in the 2018 EASL and the 2019 Chinese guidelines. In contrast, the AASLD emphasizes the application of the CT/MRI Liver Imaging Reporting and Data System (LI-RADS) (22). According to LI-RADS, the corresponding key imaging features are updated as APHE and a combination of washout, threshold growth, and capsule appearance, depending on the exact size rather than a simple combination of APHE and washout. Since 2017, contrast-enhanced ultrasound (CEUS) has been established as another diagnostic modality in China, and, in the latest EASL guidelines, 
Table 1 Comparisons of surveillance and non-invasive diagnosis of HCC among the three guidelines

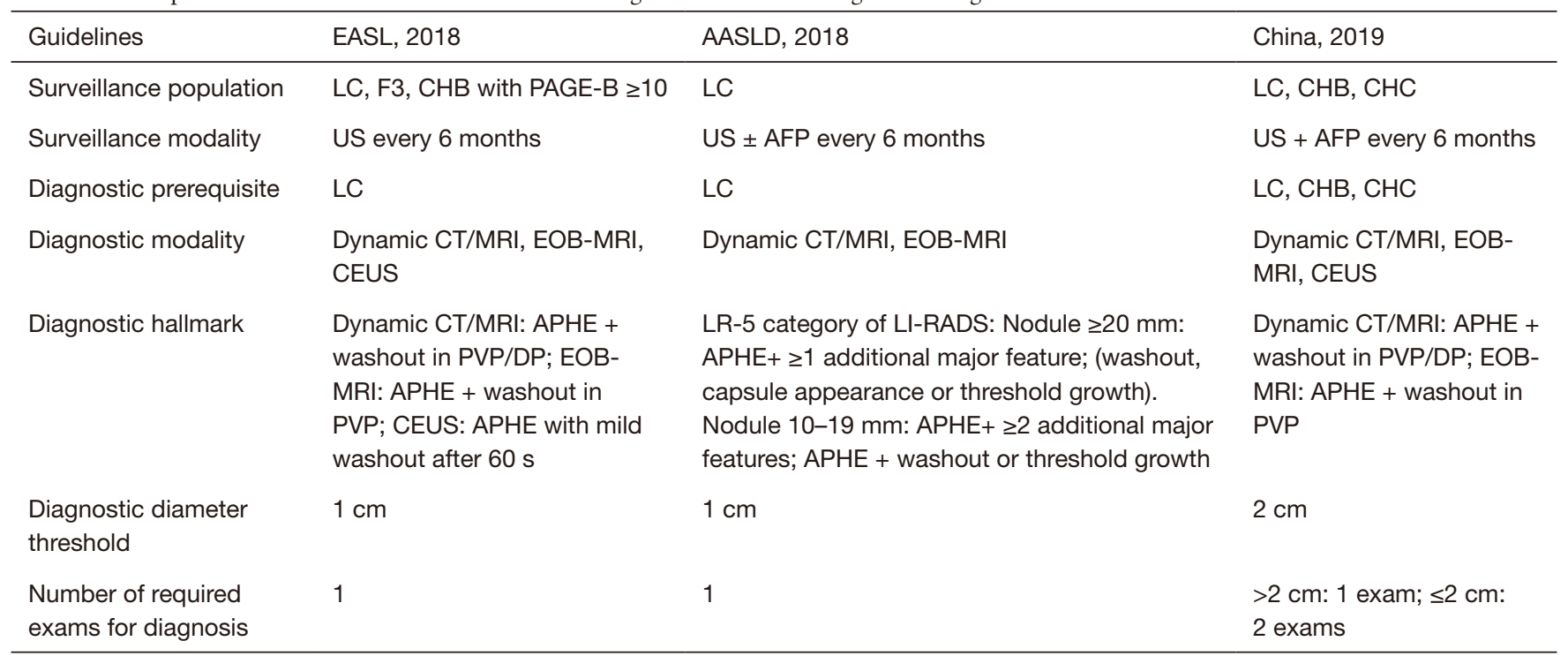

EASL, European Association for the Study of the Liver; AASLD, the American Association for the Study of Liver Diseases; LC, liver cirrhosis; F3, Metavir F3 fibrosis; CHB, chronic hepatitis B; PAGE-B (Platelet, Age, Gender, hepatitis B) score: based on decade of age (16-29 years old $=0,30-39$ years old $=2,40-49$ years old $=4,50-59$ years old $=6,60-69$ years old $=8$, $\geq 70$ years old $=10$ ), gender (male $=6$, female $=0$ ) and platelet count $(\geq 200,000 / \mu \mathrm{L}=0,100,000-199,999 / \mu \mathrm{L}=1,<100,000 / \mu \mathrm{L}=2)$; CHC, chronic hepatitis C; CT, computed tomography; MRI, magnetic resonance imaging; CEUS, contrast enhanced ultrasound; EOB-MRI, gadoxetic ethoxy benzyl diethylenetriaminepentaacetic acid-enhanced MRI; APHE, arterial phase hyperenhancement; PVP, portal vein phase; DP, delayed phase; LI-RADS, Liver Imaging Reporting and Data System.

it has been added as a second-line approach to diagnose HCC that is inconclusive on both dynamic CT and MRI. Furthermore, to discriminate it from other tumors, the typical hallmark of HCC on CEUS is APHE with lateonset $(>60 \mathrm{~s})$ washout of mild intensity $(23,24)$. However, in the latest AASLD guidelines, the application of CEUS remains restrained. The diagnostic differences of the three guidelines are summarized in Table 1.

When imaging cannot definitively determine nodules, Chinese and EASL guidelines recommend biopsy for nodules $>2$ and $>1 \mathrm{~cm}$, respectively, while the options of follow-up imaging or biopsy are deemed equally acceptable by the AASLD. The pathologic diagnoses of HCC in the current Chinese guidelines have evolved alongside improvements in therapeutic options. Besides routine staining of immunohistological markers like HSP70 (HSPA7), glypican 3 (GPC3), and glutamine synthetase (GS), programmed cell death-1(PD-1)/programmed cell death-ligand 1(PD-L1) staining is also recommended (25). Based on the KEYNOTE-028 trial, T-cell-inflamed geneexpression, PD-L1 expression, and tumor mutational burden (TMB) can predict response to PD-1 blockade in multiple tumor types (26). Histopathological features in combination with molecular assessment, such as the loss of heterozygosity, can be adopted to discriminate the clonal origin of multifocal HCCs either from intrahepatic metastasis or from multicentric occurrence in qualified centers, which may impact clinical staging and treatment allocation $(27,28)$. Novel biomarkers including liquid biopsy have shown promising diagnostic and prognostic values in various studies. A microRNA (miRNA) panel consisting of miR-122, miR-192, miR-21, miR-223, miR-26a, miR-27a, and miR-801 has demonstrated high accuracy in diagnosing early-stage $\mathrm{HBV}$-associated HCC, with an increase of sensitivity by $30 \%$ compared to AFP (29). Its commercial kit received approval from the National Medical Products Administration (NMPA) in 2017 and has been widely used in large centers in China. In addition, circulating tumor cells (CTCs) serve as a real-time parameter for monitoring treatment response. Detection of stem cell-like epithelial cell adhesion molecule (EpCAM)-positive CTCs and multi-vascular measurement of CTCs can respectively facilitate the prediction of postoperative relapse and metastasis pattern (30-32). Circulating tumor DNA (ctDNA) can also be used to identify early-stage HCC with superior sensitivity and 


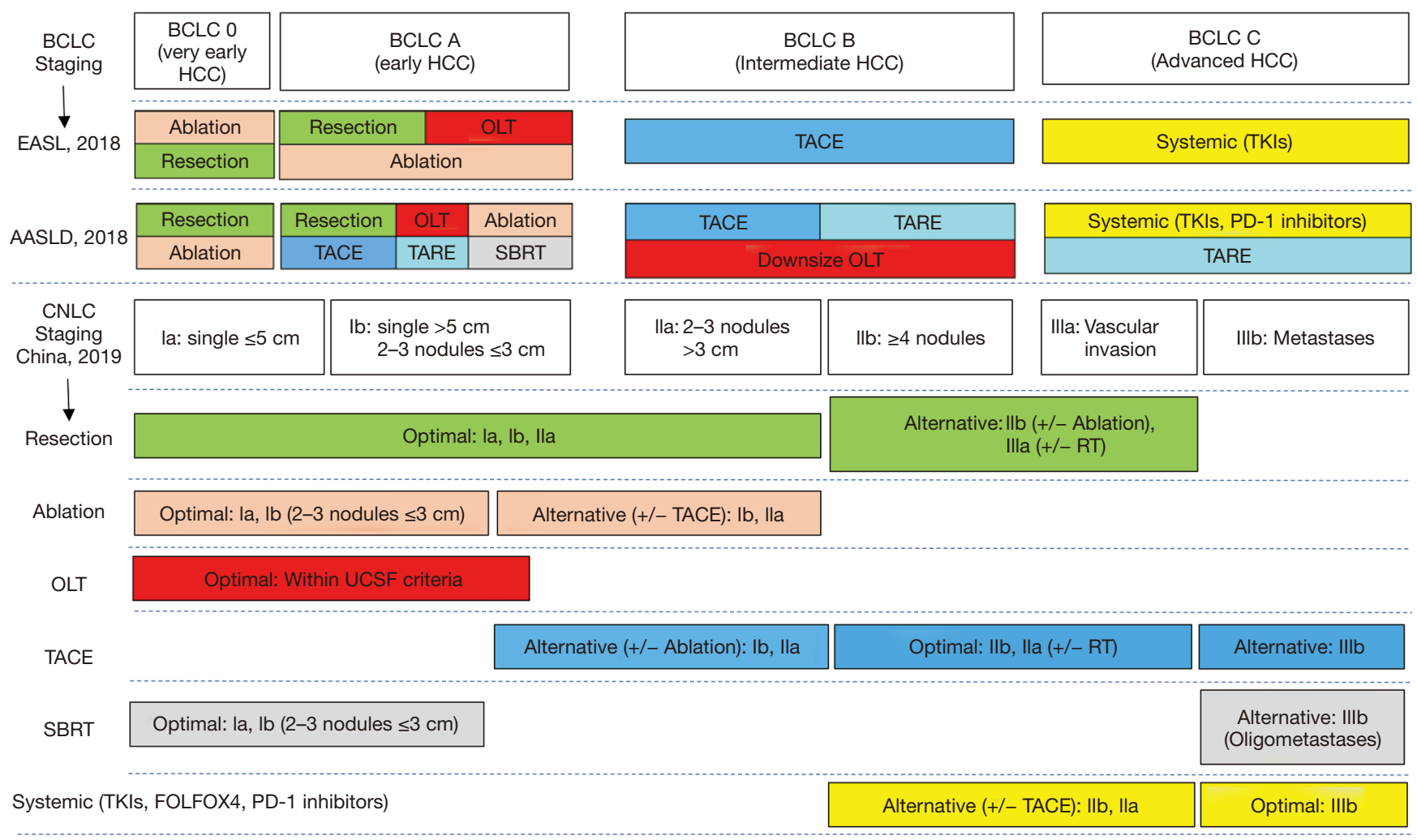

Figure 1 Comparisons of staging and treatment algorithms of HCC among 2018 EASL, 2018 AASLD, and 2019 Chinese guidelines. BCLC, Barcelona Clinic Liver Cancer; EASL, European Association for the Study of the Liver; AASLD, American Association for the Study of Liver Diseases; CNLC, China liver cancer staging; OLT, orthotopic liver transplantation; TACE, transarterial chemoembolization; TARE, transarterial radioembolization; TKIs, tyrosine kinase inhibitors; PD-1, programmed cell death-1; SBRT, stereotactic body radiation therapy; RT, radiation therapy; UCSF, University of California San Francisco; FOLFOX4, infusional fluorouracil, leucovorin, and oxaliplatin regimen.

specificity to AFP (33). The updated Chinese guidelines suggest the optional application of these new scientific findings for select patients in qualified centers.

\section{Staging and treatment allocation}

The Barcelona Clinic Liver Cancer (BCLC) classification has been extensively validated and continues to be recommended for prognostic prediction and treatment allocation by the EASL and the AASLD (34). The AASLD modifies the BCLC staging systems to better support clinical practices: the performance status (PS) scoring for BCLC stages $0, A$, and $B$ has been changed from 0 to $0-1$; and the PS for BCLC stage $\mathrm{C}$ has been expanded to $0-2$. Meanwhile, the EASL specifies the liver function for BCLC stages A-C as Child-Pugh A without ascites to obtain optimal treatment outcomes. Based on local medical systems and practice experiences, the China liver cancer staging (CNLC) system was established in 2017 and has been adopted ever since. Concerning tumor status, each stage of BCLC 0/A, B, and C is divided into two substages in the CNLC system, including stages Ia, Ib, IIa, IIb, IIIa, and IIIb. CNLC stage IV is equivalent to BCLC stage D. The updated version modifies treatment allocation to include new therapies (Figure 1). The differences in the staging systems and treatment allocations between the three guidelines are shown in Figure 1.

For patients with early-stage HCC, curative-intent options including liver resection (LR), ablation, and orthotopic liver transplantation (OLT) are determined by both anatomic considerations and liver function reserve. The AASLD recommends LR as first-line therapy for patients with BCLC 0/A HCC, whereas the EASL restricts the optimal resection criteria to single HCC. 
In Asian countries, LR is indicated for more progressed HCC in terms of tumor burden (18). Recent metaanalyses have shown a significantly improved overall survival (OS) of LR over TACE for select BCLC B and C patients $(35,36)$. The CNLC model suggests resection in patients with $\mathrm{Ia}, \mathrm{Ib}$, and IIa, and select patients with $\mathrm{IIb}$ and IIIa HCC, including multinodular HCC and locally advanced HCC with portal vein tumor thrombus (PVTT). For multinodular HCC beyond the Milan criteria, resection plus intraoperative ablation has demonstrated superior OS over TACE (37). For resectable HCC with PVTT, neoadjuvant three-dimensional (3D) conformal radiotherapy has been shown to provide significantly better survival outcomes than resection alone (38). In cases where a large volume of resection and inadequate future liver remnant (FLR) is anticipated, associating liver partition and portal vein ligation for staged hepatectomy (ALPPS) is recommended as another viable treatment option besides TACE and portal vein embolization (PVE) (39-41). Our recent propensity score matching analysis showed that the long-term survival after ALPPS was significantly better than that after TACE and similar to that after one-stage liver resection (39). Alternatively, the AASLD proposes lobar transarterial radioembolization (TARE) as an option to simultaneously treat the tumor and induce hypertrophy of the opposite lobe (42). Meanwhile, the updated Chinese guidelines advocate imaging and operative techniques that facilitate surgical resection. A new computational approach that integrates large-scale clinical and radiomic features extracted from dynamic CT/ MRI has demonstrated good performance in predicting microvascular invasion and post-operative recurrence, and is advised to be conducted before resection or transplantation $(43,44)$. The intraoperative evaluation of focal liver lesions by CEUS and shear wave elastography (SWE) enables accurate visualization and malignancy assessment of hepatic lesions $\geq 5 \mathrm{~mm}$ during liver surgery, contributing to an elevated chance of radical resection $(45,46)$. Three-dimensional (3D) visualization serves as an effective navigation tool for anatomical hepatectomy that ensures radical resection while protecting the ducts in the remnant liver $(47,48)$. The laparoscopic approach to minor hepatectomy is preferred as it confers an advantage of less blood loss while yielding similar longterm results to the open approach in experienced centers. The introduction of indocyanine green fluorescence, $3 \mathrm{D}$ laparoscopy, and robot-assisted surgery has enhanced the feasibility of laparoscopic operation, which may translate into improved surgical outcomes (49). Concerning the risk of liver function insufficiency after LR, Child-Pugh score $\mathrm{A}$, indocyanine green retention rate at $15 \mathrm{~min}$ (ICG-15) of $20-30 \%$, and residue/total liver volumetric CT of at least $40 \%$ (cirrhotic patients) or $30 \%$ (noncirrhotic patients) are listed as prerequisites for resection in the Chinese guidelines. A nomogram that integrates liver stiffness by SWE with clinic parameters may function as a valuable tool for predicting post-hepatectomy liver failure (50). Child-Pugh A and no clinically significant portal hypertension (hepatic vein to portal system gradient (HVPG) $\leq 10 \mathrm{mmHg}$ or platelet count $\geq 100,000 / \mathrm{mL}$ ) used to be the prerequisites for resection in Western countries. With evidence supporting expanded criteria for LR in cirrhosis, the EASL panel has revised the optimal candidacy to be a multiparametric evaluation including compensated Child-Pugh class A with a model for endstage liver disease (MELD) score $<10$, an acceptable grade of portal hypertension matched with a suitable amount of remaining parenchyma, and the possibility to undergo a laparoscopic/minimally invasive approach. Several studies have demonstrated that laparoscopicor robotic-assisted resections of HCC in patients with moderate portal hypertension or Child B liver function reserve are associated with decreased post-operative liver decompensation $(51,52)$. For patients with highly preoperative HBV-DNA load and normal liver function, LR can be performed with concurrent prescription of nucleotide analogues. Otherwise, it is suggested that LR be postponed until after patients have fully recovered from impaired liver function (53). While adjuvant therapy following radical resection or ablation is not recommended by the AASLD or the EASL, TACE, interferon- $\alpha$, or Huai'er granule are indicated as adjuvant options in China (54-58). For patients with intermediate risk [single nodule $>5 \mathrm{~cm}$ without microvascular invasion (MVI)] or high risk (single nodule $>5 \mathrm{~cm}$ with MVI, or multiple nodules) of recurrence, adjuvant TACE is associated with an improved OS $(54,55)$.

Despite the establishment of many extended criteria for OLT, University of California San Francisco (UCSF) criteria (solitary tumor $\leq 6.5 \mathrm{~cm}$ or $\leq 3$ nodules $\leq 4.5 \mathrm{~cm}$ plus total tumor diameter $\leq 8 \mathrm{~cm}$ ) are more widely used in China (59). Meanwhile, the EASL and the AASLD continue to recommend OLT as the first-line option for HCC within the Milan criteria (single tumor $\leq 5 \mathrm{~cm}$ or $\leq 3$ nodules $\leq 3 \mathrm{~cm}$ in diameter) in the setting of decompensated cirrhosis (60). Furthermore, the updated 
EASL and AASLD guidelines advocate bridging and downstaging via local regional therapies to increase the transplantation rate and decrease post-transplantation recurrence. Indeed, approximately $75 \%$ of recurrences following OLT occur within the first 2 years (61), while the median survival of recurrent patients is $7-16$ months with rapid progression (61). Multi-disciplinary management including immunosuppressant adjustment, graft resection, ablation, stereotactic body radiation therapy (SBRT), TACE, and systemic therapies can improve disease control and survival outcomes (62).

Thermal ablation is the standard of care for patients with BCLC 0/A tumors. While radiofrequency ablation (RFA) in favorable locations as a first-line therapy in BCLC 0 HCCs (even resectable ones) is recommended by the EASL, the superiority of resection over ablation continues to be advocated by the AASLD and Chinese experts. Imaging fusion technique combining CEUS and dynamic MRI to locate otherwise undetectable HCC on conventional ultrasound has been found to contribute to a higher ablation rate compared to CEUS guidance alone (63). The latest AASLD and Chinese guidelines have been updated to include SBRT as an alternative to ablation. Although SBRT shows comparable efficacy to RFA in several retrospective studies, comparative randomized trials are needed for confirmation (64-66). Ablation in combination with TACE has been proposed for selective inoperative solitary or multiple HCCs with a diameter of 3-7 cm in the Chinese guidelines (67); however, the survival benefit of such combinational therapy has not been validated in Western countries.

TACE continues to be recommended as the first-line therapy for intermediate BCLC B HCC by the EASL and the AASLD, whereas the CNLC model extends the indication for CNLC IIb, IIIa, and select IIIb HCC cases. A "six-and-twelve" scoring system, presented as the largest tumor diameter $(\mathrm{cm})$ plus tumor number, has proven an easy-to-use tool to stratify BCLC A/B candidates for TACE and predict individual survival with favorable performance and discrimination (68). Drug-eluting bead (DEB)-TACE is an option to minimize the systemic sideeffects of chemotherapy and has similar OS benefit as conventional TACE (cTACE) using lipiodol (69). TARE has emerged as an alternative to TACE in the updated AASLD recommendations, as it can achieve similar survival outcome with improved local tumor control compared to cTACE (70). Based on the negative results of three clinical trials, systemic agent sorafenib in combination with
TACE is not recommended by the EASL and the AASLD (71-73). However, TACE coupled with tyrosine kinase inhibitor (TKI) or immunotherapy is advocated in the latest Chinese guidelines. According to the TACTICS trial, sorafenib pretreatment $2-3$ weeks before initial TACE and continued use after TACE showed significantly improved progression-free survival (PFS) in patients with unresectable HCC (74). One reason for the competing results lies in the different pretreatment durations used in the four studies. A retrospective study has shown a promising efficacy of TACE in combination with immunotherapy in tumor control, and research into its long-term impact on survival is underway (NCT03572582, NCT03397654, NCT03143270) (75). In Western countries, TACE is considered to be a contraindication for HCC with macrovascular invasion, while China and other Asia countries recommend TACE or hepatic arterial infusion chemotherapy (HAIC) alone or in combination with other therapy for patients with portal vein invasion, even at the main trunk (76). Compared with sorafenib alone or TACE alone, TACE in combination with external beam radiation is well tolerated and results in improved local tumor control and OS for patients with macrovascular invasion (77-79); the AASLD also recognizes TARE as an additional option in this setting. It is worth noting that the current data suggest a comparable survival benefit of TARE to sorafenib in patients with locally advanced HCC $(80,81)$.

Prior to 2017, sorafenib was the only systemic treatment available for HCC, but the past 3 years have witnessed tremendous breakthroughs in this regard. Lenvatinib, boasting a comparable efficacy to sorafenib, has become another first-line option for HCC since U.S. Food and Drug Administration (FDA) and European Medicines Agency (EMA) approval was given in August 2018 (82). In a subgroup analysis of the REFLECT study, lenvatinib was superior to sorafenib (median OS 15.0 vs. 10.2 months, HR 0.73, 95\% CI: 0.55-0.96) for Chinese patients, and the NMPA approved lenvatinib as an alternative to sorafenib for patients with unresectable HCC in September 2018. In the second-line setting, two TKIs, regorafenib and cabozantinib have demonstrated survival benefit for patients with disease progression on sorafenib $(83,84)$. Since 2017 , regorafenib has been widely accepted as the first agent for second-line therapy, and, although cabozantinib is currently unavailable in China, it has become another standard second-line treatment in Western countries since the EMA and FDA gave approval in December 
2018 and January 2019 respectively. As FOLFOX4 chemotherapy has demonstrated improved OS and relative cost-effectiveness, it continues to be recommended as an option for Chinese patients (85). According to the results of REACH-2 study, ramucirumab, a monoclonal antibody targeting vascular endothelial growth factor receptor-2 (VEGFR-2), has also been established as another second-line treatment option for patients with $\mathrm{AFP} \geq 400 \mathrm{ng} / \mathrm{L}$, and will be included in the future updates (86). Apatinib, a selective VEGFR-2 TKI independently developed in China, has recently demonstrated to confer survival benefit for Chinese patients with pretreated advanced HCC and become another potential second-line agent (87). The role of immunotherapy for advanced HCC is controversial. Based on the promising results from phase II of the CHECKMATE-040 and KEYNOTE-224 trials, the AASLD has recommended PD-1 inhibitors, nivolumab and pembrolizumab, as second-line therapies for sorafenibexperienced patients $(88,89)$. Aristolochic acids exposure from Chinese herbal medicine is associated with a high tumor mutation burden and neoantigen load, which may predict better responses to immunotherapy for Chinese patients with HBV-related HCC (90). At least three native PD-1 inhibitors, including sintilimab, toripalimab, camrelizumab, are available for cancer patients in China. According to the positive results of a phase 2 study, camrelizumab has been approved by the NMPA as a secondline agent for advanced HCC (91). In contrast, the EASL has considered the evidence for a firm recommendation of this drug to be inadequate. In fact, the unmet OS benefit in the phase 3 CheckMate-459 and KEYNOTE-240 studies have stalled approval for the application of the single-agent PD-1 inhibitor in advanced HCC (92). The combination of immune checkpoint inhibitors (ICI) with TKIs is another breakthrough treatment for HCC. According to the phase 3 IMbrave150 study, the combination therapy of atezolizumab plus bevacizumab yielded superior survival over that of sorafenib, which may contribute to revisions in future guidelines regarding systemic therapies (93). Studies evaluating the efficacy of PD-1 inhibitors in combination with TKIs and chemotherapy are underway (NCT03794440, NCT03755791, NCT03713593, NCT03764293, NCT03605706, NCT03778957). Besides, the 2019 Chinese guidelines have been updated to include detailed prescriptions for traditional Chinese medicine in the treatment of advanced HCC.

\section{Conclusions}

The imaging diagnostic criteria remain consistent to the previous version in China. Novel biomarkers, imaging and operative techniques are recommended in qualified centers in the updated Chinese guideline. Compared to practices in the Western countries, more aggressive treatment modalities are suggested for more progressed HBV-related HCC in China.

\section{Future perspectives}

The availability of various imaging modalities in combination with novel biomarkers enables the diagnosis of HCC at an early stage, while the advances in the treatment modalities have reinforced a multidisciplinary approach in specialized clinics. Despite these developments, recurrence after curative therapy remains a major drawback, and more effective adjuvant therapies are needed. Identification of molecular biomarkers that predict primary or secondary resistance to TKIs or ICIs continues to be an area of intense research activity. The promising efficacy of TKIs plus ICIs may revolutionize the systemic treatment algorithm. As the number of effective systemic agents continues to grow, the challenge is to determine which order of sequential systemic therapy can offer optimal efficacy with minimal toxicity.

\section{Acknowledgments}

Funding: This work was supported by the National Natural Science Foundation of China (no. 91859105, no. 8196112802), the Basic Research Project from the Technology Commission of Shanghai Municipality (no. 17JC1402200), and the Shanghai Municipal Key Clinical Specialty.

\section{Footnote}

Reporting Checklist: The authors have completed the Narrative Review reporting checklist. Available at http:// dx.doi.org/10.21037/hbsn-20-480

Conflicts of Interest: All authors have completed the ICMJE uniform disclosure form (available at http://dx. doi. org/10.21037/hbsn-20-480). The authors have no conflicts of interest to declare. 
Ethical Statement: The authors are accountable for all aspects of the work in ensuring that questions related to the accuracy or integrity of any part of the work are appropriately investigated and resolved.

Open Access Statement: This is an Open Access article distributed in accordance with the Creative Commons Attribution-NonCommercial-NoDerivs 4.0 International License (CC BY-NC-ND 4.0), which permits the noncommercial replication and distribution of the article with the strict proviso that no changes or edits are made and the original work is properly cited (including links to both the formal publication through the relevant DOI and the license). See: https://creativecommons.org/licenses/by-nc-nd/4.0/.

\section{References}

1. Bray F, Ferlay J, Soerjomataram I, et al. Global cancer statistics 2018: GLOBOCAN estimates of incidence and mortality worldwide for 36 cancers in 185 countries. CA Cancer J Clin 2018;68:394-424.

2. Zhou $M$, Wang $\mathrm{H}$, Zeng $X$, et al. Mortality, morbidity, and risk factors in China and its provinces, 1990-2017: a systematic analysis for the Global Burden of Disease Study 2017. Lancet 2019;394:1145-58.

3. Dhanasekaran R, Nault JC, Roberts LR, et al. Genomic Medicine and Implications for Hepatocellular Carcinoma Prevention and Therapy. Gastroenterology 2019;156:492-509.

4. Llovet JM, Montal R, Sia D, et al. Molecular therapies and precision medicine for hepatocellular carcinoma. Nat Rev Clin Oncol 2018;15:599-616.

5. Pham C, Fong TL, Zhang J, et al. Striking Racial/Ethnic Disparities in Liver Cancer Incidence Rates and Temporal Trends in California, 1988-2012. J Natl Cancer Inst 2018;110:1259-69.

6. Cui F, Shen L, Li L, et al. Prevention of Chronic Hepatitis B after 3 Decades of Escalating Vaccination Policy, China. Emerg Infect Dis 2017;23:765-72.

7. Prevention of Infection Related Cancer Group SCoCP, Control CPMA, Non c, et al. Strategies of primary prevention of liver cancer in China: Expert Consensus (2018). Zhonghua Zhong Liu Za Zhi 2018;40:550-7.

8. European Association for the Study of the Liver. EASL Clinical Practice Guidelines: Management of hepatocellular carcinoma. J Hepatol 2018;69:182-236.

9. Marrero JA, Kulik LM, Sirlin CB, et al. Diagnosis, Staging, and Management of Hepatocellular
Carcinoma: 2018 Practice Guidance by the American Association for the Study of Liver Diseases. Hepatology 2018;68:723-50.

10. Zhou J, Sun HC, Wang Z, et al. Guidelines for Diagnosis and Treatment of Primary Liver Cancer in China (2017 Edition). Liver Cancer 2018;7:235-60.

11. Guidelines for Diagnosis and Treatment of Primary Liver Cancer in China (2019 Edition). Chinese Journal of Hepatology 2020:112-28.

12. Sharma SA, Kowgier M, Hansen BE, et al. Toronto HCC risk index: A validated scoring system to predict 10-year risk of HCC in patients with cirrhosis. J Hepatol 2017.

13. Zhang BH, Yang BH, Tang ZY. Randomized controlled trial of screening for hepatocellular carcinoma. J Cancer Res Clin Oncol 2004;130:417-22.

14. Papatheodoridis G, Dalekos G, Sypsa V, et al. PAGE-B predicts the risk of developing hepatocellular carcinoma in Caucasians with chronic hepatitis B on 5-year antiviral therapy. J Hepatol 2016;64:800-6.

15. Yang HI, Sherman M, Su J, et al. Nomograms for risk of hepatocellular carcinoma in patients with chronic hepatitis B virus infection. J Clin Oncol 2010;28:2437-44.

16. Yuen MF, Tanaka Y, Fong DY, et al. Independent risk factors and predictive score for the development of hepatocellular carcinoma in chronic hepatitis B. J Hepatol 2009;50:80-8.

17. Tayob N, Lok AS, Do KA, et al. Improved Detection of Hepatocellular Carcinoma by Using a Longitudinal AlphaFetoprotein Screening Algorithm. Clin Gastroenterol Hepatol 2016;14:469-75 e2.

18. Omata M, Cheng AL, Kokudo N, et al. Asia-Pacific clinical practice guidelines on the management of hepatocellular carcinoma: a 2017 update. Hepatol Int 2017;11:317-70.

19. Liu X, Jiang H, Chen J, et al. Gadoxetic acid disodiumenhanced magnetic resonance imaging outperformed multidetector computed tomography in diagnosing small hepatocellular carcinoma: A meta-analysis. Liver Transpl 2017;23:1505-18.

20. Li J, Wang J, Lei L, et al. The diagnostic performance of gadoxetic acid disodium-enhanced magnetic resonance imaging and contrast-enhanced multi-detector computed tomography in detecting hepatocellular carcinoma: a meta-analysis of eight prospective studies. Eur Radiol 2019;29:6519-28.

21. Aube C, Oberti F, Lonjon J, et al. EASL and AASLD recommendations for the diagnosis of HCC to the test of daily practice. Liver Int 2017;37:1515-25. 
22. Tang A, Bashir MR, Corwin MT, et al. Evidence Supporting LI-RADS Major Features for CT- and MR Imaging-based Diagnosis of Hepatocellular Carcinoma: A Systematic Review. Radiology 2018;286:29-48.

23. Piscaglia F, Wilson SR, Lyshchik A, et al. American College of Radiology Contrast Enhanced Ultrasound Liver Imaging Reporting and Data System (CEUS LIRADS) for the diagnosis of Hepatocellular Carcinoma: a pictorial essay. Ultraschall Med 2017;38:320-4.

24. Terzi E, Iavarone M, Pompili M, et al. Contrast ultrasound LI-RADS LR-5 identifies hepatocellular carcinoma in cirrhosis in a multicenter restropective study of 1,006 nodules. J Hepatol 2018;68:485-92.

25. Shi L, Zhang SJ, Chen J, et al. A comparability study of immunohistochemical assays for PD-L1 expression in hepatocellular carcinoma. Mod Pathol 2019;32:1646-56.

26. Ott PA, Bang YJ, Piha-Paul SA, et al. T-Cell-Inflamed Gene-Expression Profile, Programmed Death Ligand 1 Expression, and Tumor Mutational Burden Predict Efficacy in Patients Treated With Pembrolizumab Across 20 Cancers: KEYNOTE-028. J Clin Oncol 2019;37:318-27.

27. Xie DY, Fan HK, Ren ZG, et al. Identifying Clonal Origin of Multifocal Hepatocellular Carcinoma and Its Clinical Implications. Clin Transl Gastroenterol 2019;10:e00006.

28. Wen T, Jin C, Facciorusso A, et al. Multidisciplinary management of recurrent and metastatic hepatocellular carcinoma after resection: an international expert consensus. Hepatobiliary Surg Nutr 2018;7:353-71.

29. Zhou J, Yu L, Gao X, et al. Plasma microRNA panel to diagnose hepatitis $\mathrm{B}$ virus-related hepatocellular carcinoma. J Clin Oncol 2011;29:4781-8.

30. Sun YF, Xu Y, Yang XR, et al. Circulating stem cell-like epithelial cell adhesion molecule-positive tumor cells indicate poor prognosis of hepatocellular carcinoma after curative resection. Hepatology 2013;57:1458-68.

31. Sun YF, Guo W, Xu Y, et al. Circulating Tumor Cells from Different Vascular Sites Exhibit Spatial Heterogeneity in Epithelial and Mesenchymal Composition and Distinct Clinical Significance in Hepatocellular Carcinoma. Clin Cancer Res 2018;24:547-59.

32. Guo W, Yang XR, Sun YF, et al. Clinical significance of EpCAM mRNA-positive circulating tumor cells in hepatocellular carcinoma by an optimized negative enrichment and qRT-PCR-based platform. Clin Cancer Res 2014;20:4794-805.

33. Qu C, Wang Y, Wang P, et al. Detection of early-stage hepatocellular carcinoma in asymptomatic HBsAgseropositive individuals by liquid biopsy. Proc Natl Acad Sci U S A 2019;116:6308-12.

34. Cillo U, Vitale A, Grigoletto F, et al. Prospective validation of the Barcelona Clinic Liver Cancer staging system. J Hepatol 2006;44:723-31.

35. Hyun MH, Lee YS, Kim JH, et al. Hepatic resection compared to chemoembolization in intermediate- to advanced-stage hepatocellular carcinoma: A meta-analysis of high-quality studies. Hepatology 2018;68:977-93.

36. Tsilimigras DI, Bagante F, Moris D, et al. Recurrence Patterns and Outcomes after Resection of Hepatocellular Carcinoma within and beyond the Barcelona Clinic Liver Cancer Criteria. Ann Surg Oncol 2020;27:2321-31.

37. Zhou C, Peng Y, Zhou K, et al. Surgical resection plus radiofrequency ablation for the treatment of multifocal hepatocellular carcinoma. Hepatobiliary Surg Nutr 2019;8:19-28.

38. Wei X, Jiang Y, Zhang X, et al. Neoadjuvant ThreeDimensional Conformal Radiotherapy for Resectable Hepatocellular Carcinoma With Portal Vein Tumor Thrombus: A Randomized, Open-Label, Multicenter Controlled Study. J Clin Oncol 2019;37:2141-51.

39. Wang Z, Peng Y, Hu J, et al. Associating Liver Partition and Portal Vein Ligation for Staged Hepatectomy for Unresectable Hepatitis B Virus-related Hepatocellular Carcinoma: A Single Center Study of 45 Patients. Ann Surg 2020;271:534-41.

40. Glantzounis GK, Tokidis E, Basourakos SP, et al. The role of portal vein embolization in the surgical management of primary hepatobiliary cancers. A systematic review. Eur J Surg Oncol 2017;43:32-41.

41. Li C, Wang MD, Lu L, et al. Preoperative transcatheter arterial chemoembolization for surgical resection of huge hepatocellular carcinoma $(>/=10 \mathrm{~cm})$ : a multicenter propensity matching analysis. Hepatol Int 2019;13:736-47.

42. Theysohn JM, Ertle J, Muller S, et al. Hepatic volume changes after lobar selective internal radiation therapy (SIRT) of hepatocellular carcinoma. Clin Radiol 2014;69:172-8.

43. Xu X, Zhang HL, Liu QP, et al. Radiomic analysis of contrast-enhanced CT predicts microvascular invasion and outcome in hepatocellular carcinoma. J Hepatol 2019;70:1133-44.

44. Lei Z, Li J, Wu D, et al. Nomogram for Preoperative Estimation of Microvascular Invasion Risk in Hepatitis B Virus-Related Hepatocellular Carcinoma Within the 
Milan Criteria. JAMA Surg 2016;151:356-63.

45. da Silva NPB, Hornung M, Beyer LP, et al. Intraoperative Shear Wave Elastography vs. Contrast-Enhanced Ultrasound for the Characterization and Differentiation of Focal Liver Lesions to Optimize Liver Tumor Surgery. Ultraschall Med 2019;40:205-11.

46. Grgurevic I, Bokun T, Salkic NN, et al. Liver elastography malignancy prediction score for noninvasive characterization of focal liver lesions. Liver Int 2018;38:1055-63.

47. Mise Y, Hasegawa K, Satou S, et al. How Has Virtual Hepatectomy Changed the Practice of Liver Surgery? Experience of 1194 Virtual Hepatectomy Before Liver Resection and Living Donor Liver Transplantation. Ann Surg 2018;268:127-33.

48. Yang J, Tao HS, Cai W, et al. Accuracy of actual resected liver volume in anatomical liver resections guided by 3 -dimensional parenchymal staining using fusion indocyanine green fluorescence imaging. J Surg Oncol 2018;118:1081-7.

49. Cheung TT, Han HS, She WH, et al. The Asia Pacific Consensus Statement on Laparoscopic Liver Resection for Hepatocellular Carcinoma: A Report from the 7th AsiaPacific Primary Liver Cancer Expert Meeting Held in Hong Kong. Liver Cancer 2018;7:28-39.

50. Hu H, Han H, Han XK, et al. Nomogram for individualised prediction of liver failure risk after hepatectomy in patients with resectable hepatocellular carcinoma: the evidence from ultrasound data. Eur Radiol 2018;28:877-85.

51. Han HS, Shehta A, Ahn S, et al. Laparoscopic versus open liver resection for hepatocellular carcinoma: Casematched study with propensity score matching. J Hepatol 2015;63:643-50.

52. Sposito C, Battiston C, Facciorusso A, et al. Propensity score analysis of outcomes following laparoscopic or open liver resection for hepatocellular carcinoma. Br J Surg 2016;103:871-80.

53. Huang G, Lau WY, Wang ZG, et al. Antiviral therapy improves postoperative survival in patients with hepatocellular carcinoma: a randomized controlled trial. Ann Surg 2015;261:56-66.

54. Wang Z, Ren Z, Chen Y, et al. Adjuvant Transarterial Chemoembolization for HBV-Related Hepatocellular Carcinoma After Resection: A Randomized Controlled Study. Clin Cancer Res 2018;24:2074-81.

55. Wei W, Jian PE, Li SH, et al. Adjuvant transcatheter arterial chemoembolization after curative resection for hepatocellular carcinoma patients with solitary tumor and microvascular invasion: a randomized clinical trial of efficacy and safety. Cancer Commun (Lond) 2018;38:61.

56. Chen Q, Shu C, Laurence AD, et al. Effect of Huaier granule on recurrence after curative resection of HCC: a multicentre, randomised clinical trial. Gut 2018;67:2006-16.

57. Bruix J, Takayama T, Mazzaferro V, et al. Adjuvant sorafenib for hepatocellular carcinoma after resection or ablation (STORM): a phase 3, randomised, double-blind, placebo-controlled trial. Lancet Oncol 2015;16:1344-54.

58. Lo CM, Liu CL, Chan SC, et al. A randomized, controlled trial of postoperative adjuvant interferon therapy after resection of hepatocellular carcinoma. Ann Surg 2007;245:831-42.

59. Yao FY, Ferrell L, Bass NM, et al. Liver transplantation for hepatocellular carcinoma: expansion of the tumor size limits does not adversely impact survival. Hepatology 2001;33:1394-403.

60. Clavien PA, Lesurtel M, Bossuyt PM, et al. Recommendations for liver transplantation for hepatocellular carcinoma: an international consensus conference report. Lancet Oncol 2012;13:e11-22.

61. Filgueira NA. Hepatocellular carcinoma recurrence after liver transplantation: Risk factors, screening and clinical presentation. World J Hepatol 2019;11:261-72.

62. Au KP, Chok KSH. Multidisciplinary approach for postliver transplant recurrence of hepatocellular carcinoma: A proposed management algorithm. World J Gastroenterol 2018;24:5081-94

63. Dong Y, Wang WP, Mao F, et al. Application of imaging fusion combining contrast-enhanced ultrasound and magnetic resonance imaging in detection of hepatic cellular carcinomas undetectable by conventional ultrasound. J Gastroenterol Hepatol 2016;31:822-8.

64. Kim N, Cheng J, Jung I, et al. Stereotactic body radiation therapy vs. radiofrequency ablation in Asian patients with hepatocellular carcinoma. J Hepatol 2020.

65. Wahl DR, Stenmark MH, Tao Y, et al. Outcomes After Stereotactic Body Radiotherapy or Radiofrequency Ablation for Hepatocellular Carcinoma. J Clin Oncol 2016;34:452-9.

66. Hara K, Takeda A, Tsurugai Y, et al. Radiotherapy for Hepatocellular Carcinoma Results in Comparable Survival to Radiofrequency Ablation: A Propensity Score Analysis. 
Hepatology 2019;69:2533-45.

67. Pan T, Mu LW, Wu C, et al. Comparison of Combined Transcatheter Arterial Chemoembolization and CTguided Radiofrequency Ablation with Surgical Resection in Patients with Hepatocellular Carcinoma within the Upto-seven Criteria: A Multicenter Case-matched Study. J Cancer 2017;8:3506-13.

68. Wang Q, Xia D, Bai W, et al. Development of a prognostic score for recommended TACE candidates with hepatocellular carcinoma: A multicentre observational study. J Hepatol 2019;70:893-903.

69. Ma Y, Zhao C, Zhao H, et al. Comparison of treatment efficacy and safety between drug-eluting bead transarterial chemoembolization with CalliSpheres((R)) microspheres and conventional transarterial chemoembolization as firstline treatment in hepatocellular carcinoma patients. Am J Transl Res 2019;11:7456-70.

70. Casadei Gardini A, Tamburini E, Inarrairaegui M, et al. Radioembolization versus chemoembolization for unresectable hepatocellular carcinoma: a metaanalysis of randomized trials. Onco Targets Ther 2018;11:7315-21.

71. Kudo M, Imanaka K, Chida N, et al. Phase III study of sorafenib after transarterial chemoembolisation in Japanese and Korean patients with unresectable hepatocellular carcinoma. Eur J Cancer 2011;47:2117-27.

72. Lencioni R, Llovet JM, Han G, et al. Sorafenib or placebo plus TACE with doxorubicin-eluting beads for intermediate stage HCC: The SPACE trial. J Hepatol 2016;64:1090-8.

73. Meyer T, Fox R, Ma YT, et al. Sorafenib in combination with transarterial chemoembolisation in patients with unresectable hepatocellular carcinoma (TACE 2): a randomised placebo-controlled, double-blind, phase 3 trial. Lancet Gastroenterol Hepatol 2017;2:565-75.

74. Kudo M, Ueshima K, Ikeda M, et al. Randomised, multicentre prospective trial of transarterial chemoembolisation (TACE) plus sorafenib as compared with TACE alone in patients with hepatocellular carcinoma: TACTICS trial. Gut 2020;69:1492-501

75. Duffy AG, Ulahannan SV, Makorova-Rusher O, et al. Tremelimumab in combination with ablation in patients with advanced hepatocellular carcinoma. J Hepatol 2017;66:545-51.

76. Kudo M, Matsui O, Izumi N, et al. JSH ConsensusBased Clinical Practice Guidelines for the Management of Hepatocellular Carcinoma: 2014 Update by the Liver Cancer Study Group of Japan.
Liver Cancer 2014;3:458-68.

77. Yoon SM, Ryoo BY, Lee SJ, et al. Efficacy and Safety of Transarterial Chemoembolization Plus External Beam Radiotherapy vs Sorafenib in Hepatocellular Carcinoma With Macroscopic Vascular Invasion: A Randomized Clinical Trial. JAMA Oncol 2018;4:661-9.

78. Shen L, Xi M, Zhao L, et al. Combination Therapy after TACE for Hepatocellular Carcinoma with Macroscopic Vascular Invasion: Stereotactic Body Radiotherapy versus Sorafenib. Cancers (Basel) 2018;10:516.

79. Huo YR, Eslick GD. Transcatheter Arterial Chemoembolization Plus Radiotherapy Compared With Chemoembolization Alone for Hepatocellular Carcinoma: A Systematic Review and Meta-analysis. JAMA Oncol 2015;1:756-65.

80. Chow PKH, Gandhi M, Tan SB, et al. SIRveNIB: Selective Internal Radiation Therapy Versus Sorafenib in Asia-Pacific Patients With Hepatocellular Carcinoma. J Clin Oncol 2018;36:1913-21.

81. Vilgrain V, Pereira H, Assenat E, et al. Efficacy and safety of selective internal radiotherapy with yttrium-90 resin microspheres compared with sorafenib in locally advanced and inoperable hepatocellular carcinoma (SARAH): an open-label randomised controlled phase 3 trial. Lancet Oncol 2017;18:1624-36.

82. Kudo M, Finn RS, Qin S, et al. Lenvatinib versus sorafenib in first-line treatment of patients with unresectable hepatocellular carcinoma: a randomised phase 3 noninferiority trial. Lancet 2018;391:1163-73.

83. Finn RS, Merle P, Granito A, et al. Outcomes of sequential treatment with sorafenib followed by regorafenib for HCC: Additional analyses from the phase III RESORCE trial. J Hepatol 2018;69:353-8.

84. Abou-Alfa GK, Meyer T, Cheng AL, et al. Cabozantinib in Patients with Advanced and Progressing Hepatocellular Carcinoma. N Engl J Med 2018;379:54-63.

85. Qin S, Bai Y, Lim HY, et al. Randomized, multicenter, open-label study of oxaliplatin plus fluorouracil/leucovorin versus doxorubicin as palliative chemotherapy in patients with advanced hepatocellular carcinoma from Asia. J Clin Oncol 2013;31:3501-8.

86. Zhu AX, Kang YK, Yen CJ, et al. Ramucirumab after sorafenib in patients with advanced hepatocellular carcinoma and increased alpha-fetoprotein concentrations (REACH-2): a randomised, double-blind, placebocontrolled, phase 3 trial. Lancet Oncol 2019;20:282-96.

87. Yang C, Qin S. Apatinib targets both tumor and endothelial cells in hepatocellular carcinoma. Cancer Med 
2018;7:4570-83.

88. El-Khoueiry AB, Sangro B, Yau T, et al. Nivolumab in patients with advanced hepatocellular carcinoma (CheckMate 040): an open-label, non-comparative, phase 1/2 dose escalation and expansion trial. Lancet 2017;389:2492-502.

89. Zhu AX, Finn RS, Edeline J, et al. Pembrolizumab in patients with advanced hepatocellular carcinoma previously treated with sorafenib (KEYNOTE-224): a non-randomised, open-label phase 2 trial. Lancet Oncol 2018;19:940-52.

90. Gao Q, Zhu H, Dong L, et al. Integrated Proteogenomic Characterization of HBV-Related Hepatocellular Carcinoma. Cell 2019;179:561-77.

Cite this article as: Xie DY, Ren ZG, Zhou J, Fan J, Gao Q. 2019 Chinese clinical guidelines for the management of hepatocellular carcinoma: updates and insights. HepatoBiliary Surg Nutr 2020;9(4):452-463. doi: 10.21037/hbsn-20-480
91. Qin S, Ren Z, Meng Z, et al. Camrelizumab in patients with previously treated advanced hepatocellular carcinoma: a multicentre, open-label, parallel-group, randomised, phase 2 trial. Lancet Oncol 2020;21:571-80.

92. Finn RS, Ryoo BY, Merle P, et al. Pembrolizumab As Second-Line Therapy in Patients With Advanced Hepatocellular Carcinoma in KEYNOTE-240: A Randomized, Double-Blind, Phase III Trial. J Clin Oncol 2020;38:193-202.

93. Finn RS, Qin S, Ikeda M, et al. Atezolizumab plus Bevacizumab in Unresectable Hepatocellular Carcinoma. N Engl J Med 2020;382:1894-905.

(English Language Editor: J. Gray) 